\title{
Research on Radial Vibration of a Circular Plate
}

\author{
Wei Liu, Donghua Wang, Hanfeng Lu, Yumeng Cao, and Pengrong Zhang
}

College of Power and Energy Engineering, Harbin Engineering University, Harbin, China

Correspondence should be addressed to Donghua Wang; hitwdh@163.com

Received 25 May 2016; Revised 13 September 2016; Accepted 29 September 2016

Academic Editor: Giorgio Dalpiaz

Copyright (C) 2016 Wei Liu et al. This is an open access article distributed under the Creative Commons Attribution License, which permits unrestricted use, distribution, and reproduction in any medium, provided the original work is properly cited.

Radial vibration of the circular plate is presented using wave propagation approach and classical method containing Bessel solution and Hankel solution for calculating the natural frequency theoretically. In cylindrical coordinate system, in order to obtain natural frequency, propagation and reflection matrices are deduced at the boundaries of free-free, fixed-fixed, and fixed-free using wave propagation approach. Furthermore, radial phononic crystal is constructed by connecting two materials periodically for the analysis of band phenomenon. Also, Finite Element Simulation (FEM) is adopted to verify the theoretical results. Finally, the radial and piezoelectric effects on the band are also discussed.

\section{Introduction}

Wave propagation approach is a useful method on the aspects of calculating natural frequency for the analysis of elastic structures, such as rod, Euler beam, curved beam, and plate. It benefits us with a better understanding to analyze wave when propagating inside structures. In fact, as early as 1984, Mace analyzed the behaviors of wave by using wave propagation approach through dividing them into propagation attenuation matrices. And the reflection matrices under three boundary conditions were deduced, which built a theoretical foundation for wave method [1]. Meanwhile, the characteristics of propagation and reflection of Timoshenko beam were also discussed with the discontinuous behavior. And the frequency curves were obtained [2]. Harland et al. studied the motion of waves in tunable fluid-filled beams [3]. Based on Love theory, Kang et al. deduced the propagation matrices, reflection matrices, and coordinated matrices in curved beams [4]. Different from Kang et al., Lee made a detailed research on the behavior of waves of curved beam based on Flugge's theory [5, 6]. Based on the high-order plate theory, Wang and Rose analyzed the properties of transient wave in an inhomogeneous beam. And the results were also verified by the experiment [7]. Tan and Kang considered the wave reflection and transmission in an axially strained, rotating Timoshenko shaft. Meanwhile, the influence of continuous condition and cross-section on natural frequency was also discussed [8]. Li and Gan analyzed the free vibration of cylindrical shell by using wave propagation approach based on Flugge's thin shell theory $[9,10]$. Also, Zhang calculated the frequencies of cross-ply laminated composite cylindrical shells and submerged cylindrical shells using wave approach based on Love's theory [11, 12]. Using wave approach, the above scholars have done lots of analysis, which exhibit a unique advantage for the analysis of structural vibration.

In recent years, people have paid great attention to structure which consisted of two materials arraying periodically. At present, the research has been extended into radial phononic crystal structures. Shakeri et al. analyzed the behaviors of radial wave in functional graded radial periodical structures [13]. Torrent and Carbonell studied the propagation characteristic of acoustic in detail when propagating in radial periodical structure and found that it also had a low frequency band $[14,15]$. After constructing and analyzing the two-dimensional radial phononic crystal, $\mathrm{Xu}$ et al. found that these structures had low frequency band that the starting frequency was zero [16]. Ma et al. studied the band characteristics of Lamb wave in two-layer radial phononic crystal plate, and it was found that crystal gliding in radial direction could open the lowest band gaps [17]. The above researches indicate that radial phononic crystal plate has the low frequency band which brings an attractive prospect of engineering application. For example, the natural frequency can be designed into the range of band gaps to 


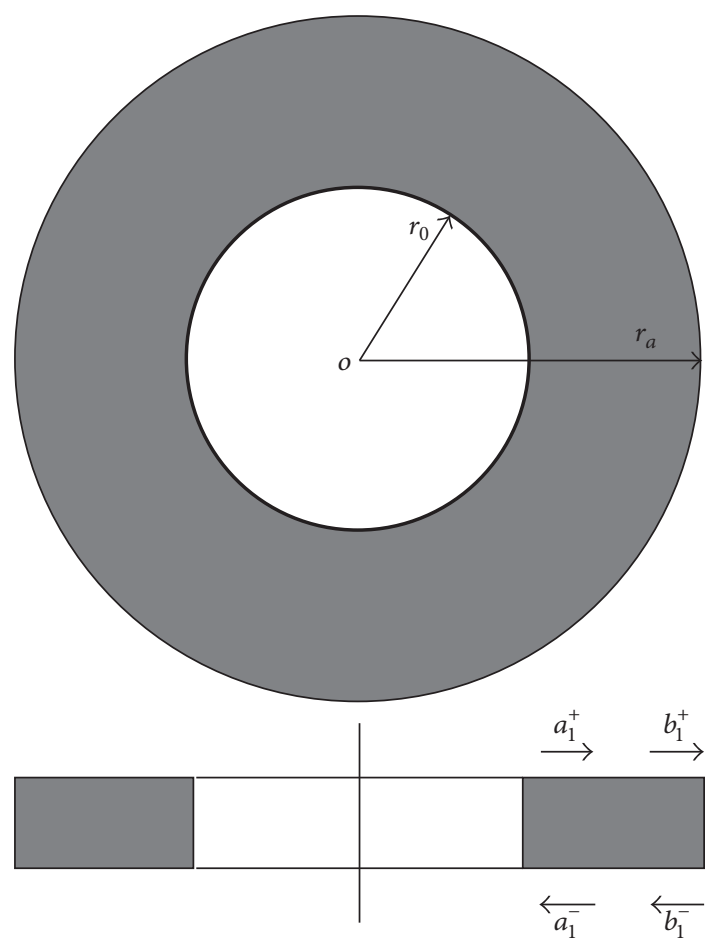

FIgURE 1: Model of single circular plate.

isolate the vibration. Also, these structures can be regarded as a filter. Additionally, Shu et al. researched torsional vibration problem of generalized phononic crystal, finding that low frequency band was determined by radius. However, the high frequency band was caused by the periodicity of circular plate [18]. And Shu's research shows that generalized phononic crystal can generate a huge band which almost covers the whole frequency range, providing a practical application for the rotating parts, such as gear. In the previous research, Shu et al. have studied the band gaps of flexural wave in the radial phononic crystal. Meanwhile, numerical results are also verified by FEM [19]. Herein, this paper is mainly focused on radial vibration of composite structures. In fact, other works on radial vibration of elastic solids under various loadings can also be found [20-22].

The paper is organized into five sections. In Section 1 , a brief introduction is given. In Section 2, classic Bessel solution and Hankel solution of radial vibration are given. Meanwhile, propagation and reflection matrices at three boundary conditions are derived. The results of natural frequency obtained by wave approach are also compared with the classical method. In Section 3, the band phenomenon is analyzed and the effect of parameters is discussed. Section 4 is the conclusion.

\section{Theoretical Analysis for Natural Frequency}

2.1. Solution of Radial Vibration Equation. Single circular plate is shown in Figure 1. Young modulus, density, and Poisson's coefficient are $E_{1}, \rho_{1}, \nu_{1}$. The thickness of piezoelectric ceramics is $h_{1}$. The inner and external radius are $r_{0}$ and $r_{a}$, respectively. With respect to the axisymmetric circular plates, radial equation of piezoelectric materials can be given by [23]

$$
\rho \frac{\partial^{2} u_{r}}{\partial t^{2}}=\frac{\partial \sigma_{r}}{\partial r}+\frac{\sigma_{r}-\sigma_{\theta}}{r} .
$$

The expression of strain and radial displacement can be written as

$$
\begin{gathered}
\varepsilon_{r}=\frac{\partial u_{r}}{\partial r}, \\
\varepsilon_{\theta}=\frac{u_{r}}{r} .
\end{gathered}
$$

Since piezoelectric material direction of polarization is along $z$-axle, piezoelectric equation can be expressed as

$$
\begin{gathered}
\varepsilon_{r}=s_{11}^{E} \sigma_{r}+s_{12}^{E} \sigma_{\theta}+d_{31} E_{Z}, \\
\varepsilon_{\theta}=s_{12}^{E} \sigma_{r}+s_{11}^{E} \sigma_{\theta}+d_{31} E_{Z}, \\
D_{z}=d_{31} \sigma_{r}+d_{31} \sigma_{\theta}+\xi_{33}^{T} E_{Z},
\end{gathered}
$$

where $u_{r}$ is radial displacement, $\sigma_{r}$ and $\sigma_{\theta}$ are radial stress and tangential stress, $\varepsilon_{r}$ and $\varepsilon_{\theta}$ are radial strain and tangential strain, and $r$ is radius.

Substituting (2)-(4) into (1) gives

$$
\frac{\partial^{2} u_{r}}{\partial r^{2}}+\frac{1}{r} \frac{\partial u_{r}}{\partial r}-\frac{u_{r}}{r^{2}}=\frac{1}{c^{2}} \frac{\partial^{2} u_{r}}{\partial t^{2}},
$$

where $c^{2}=1 / s_{11}^{E} \rho\left(1-v^{2}\right)$ is the square of radial wave velocity.

After simplifying (5), it can be obtained that

$$
\frac{\partial^{2} u_{r}}{\partial(k r)^{2}}+\frac{1}{k r} \frac{\partial u_{r}}{\partial(k r)}+\left[1-\frac{1}{(k r)^{2}}\right] u=0 .
$$

Thus, general solution of radial displacement can be written as

$$
u_{r}=A_{1}^{+} J_{1}(k r)+A_{1}^{-} Y_{1}(k r)
$$

where $k=w / c$ is the wave number. $J_{1}(k r)$ is the Bessel function of first kind; $Y_{1}(k r)$ is the Bessel function of second kind. And the constants $A_{1}^{+}$and $A_{1}^{-}$can be determined by boundary conditions.

Therefore, radial stress can be obtained as

$$
\begin{aligned}
\sigma_{r} & =\frac{E}{1-v^{2}}\left(\varepsilon_{r}+\nu \varepsilon_{\theta}\right) \\
& =\frac{E k}{1-v^{2}}\left\{A_{1}^{+}\left[J_{0}(k r)-\frac{(1-v) J_{1}(k r)}{k r}\right]\right. \\
& \left.+A_{1}^{-}\left[Y_{0}(k r)-\frac{(1-v) Y_{1}(k r)}{k r}\right]\right\} .
\end{aligned}
$$

2.2. Classical Method. When both ends of circular plate are fixed, it has

$$
\begin{aligned}
& u_{r}\left(r_{0}\right)=0, \\
& u_{r}\left(r_{a}\right)=0 .
\end{aligned}
$$


Substituting (7) into (9) gives

$$
\left|\begin{array}{lll}
J_{1}\left(k r_{0}\right) & Y_{1}\left(k r_{0}\right) \\
J_{1}\left(k r_{a}\right) & Y_{1}\left(k r_{a}\right)
\end{array}\right|=0 .
$$

When both ends of circular plate are free, it has

$$
\begin{aligned}
& \sigma_{r}\left(r_{0}\right)=0, \\
& \sigma_{r}\left(r_{a}\right)=0 .
\end{aligned}
$$

Substituting (8) into (11) gives

$$
\begin{aligned}
& \frac{E k}{1-v^{2}} \\
& \qquad\left|\begin{array}{ll}
J_{0}\left(k r_{0}\right)-\frac{(1-v) J_{1}\left(k r_{0}\right)}{k r_{0}} & Y_{0}\left(k r_{0}\right)-\frac{(1-v) Y_{1}\left(k r_{0}\right)}{k r_{0}} \\
J_{0}\left(k r_{a}\right)-\frac{(1-v) J_{1}\left(k r_{a}\right)}{k r_{a}} & J_{0}\left(k r_{a}\right)-\frac{(1-v) J_{1}\left(k r_{a}\right)}{k r_{a}}
\end{array}\right| \\
& \quad=0 .
\end{aligned}
$$

Similarly, when inner surface is fixed and outer surface is free, it has

$$
\begin{aligned}
& u_{r}\left(r_{0}\right)=0, \\
& \sigma_{r}\left(r_{a}\right)=0 .
\end{aligned}
$$

Substituting (7) and (8) into (13) gives

$$
\begin{aligned}
& \frac{E k}{1-v^{2}} \\
& \cdot\left|\begin{array}{cc}
J_{0}\left(k r_{0}\right) & Y_{0}\left(k r_{0}\right) \\
J_{0}\left(k r_{a}\right)-\frac{(1-\nu) J_{1}\left(k r_{a}\right)}{k r_{a}} & J_{0}\left(k r_{a}\right)-\frac{(1-v) J_{1}\left(k r_{a}\right)}{k r_{a}}
\end{array}\right| \\
& =0 .
\end{aligned}
$$

After solving roots of (10), (12), and (14), the natural frequencies of circular plate structure can be obtained with the aforementioned boundary conditions.

2.3. Wave Propagation Method. One of the solutions of circular plate has been solved as shown in (7). And the other solution can be written as

$$
u_{r}=A_{1}^{+} H_{0}^{(2)}(k r)+A_{1}^{-} H_{0}^{(1)}(k r),
$$

where $H_{0}^{(1)}(k r)$ and $H_{0}^{(2)}(k r)$ are the Hankel function of first and second kinds, which can be defined as

$$
\begin{aligned}
& H_{0}^{(1)}(k r)=J_{0}(k r)+i Y_{0}(k r), \\
& H_{0}^{(2)}(k r)=J_{0}(k r)-i Y_{0}(k r) .
\end{aligned}
$$

Considering (15), forward-traveling and negativetraveling waves can be written as

$$
\begin{aligned}
& g_{1}^{+}=A_{1}^{+} H_{0}^{(2)}(k r), \\
& g_{1}^{-}=A_{1}^{-} H_{0}^{(1)}(k r) .
\end{aligned}
$$

Then, (15) reduces to

$$
u_{r}=g_{1}^{+}+g_{1}^{-} .
$$

When both ends of circular plate are fixed, it gives

$$
\begin{aligned}
& u_{r}\left(r_{0}\right)=g_{1}^{+}\left(r_{0}\right)+g_{1}^{-}\left(r_{0}\right)=0, \\
& u_{r}\left(r_{a}\right)=g_{1}^{+}\left(r_{a}\right)+g_{1}^{-}\left(r_{a}\right)=0 .
\end{aligned}
$$

Thus, the reflection matrices of both boundaries are

$$
\begin{aligned}
& R_{A}=[-1], \\
& R_{C}=[-1] .
\end{aligned}
$$

Substituting (2), (15), and (16) into (8), radial stress can be expressed as

$$
\begin{aligned}
\sigma_{r} & =\frac{E k}{1-v^{2}}\left\{A _ { 1 } ^ { + } \left[k J_{0}(k r)-\frac{J_{1}(k r)}{r}-i k Y_{0}(k r)\right.\right. \\
& \left.+\frac{i}{r} Y_{1}(k r)+\frac{v}{r} J_{1}(k r)-\frac{v}{r} i Y_{1}(k r)\right]+A_{1}^{-}\left[k J_{0}(k r)\right. \\
& -\frac{J_{1}(k r)}{r}+i k Y_{0}(k r)-\frac{i}{r} Y_{1}(k r)+\frac{v}{r} J_{1}(k r) \\
& \left.\left.+\frac{v}{r} i Y_{1}(k r)\right]\right\} .
\end{aligned}
$$

When both ends of circular plate are free, it has

$$
\begin{aligned}
& \sigma_{r}\left(r_{0}\right)=0, \\
& \sigma_{r}\left(r_{a}\right)=0 .
\end{aligned}
$$

Thus, reflection matrices of both boundaries are

$$
\begin{aligned}
R_{A} & =-\left\{\frac{\left[k J_{0}\left(k r_{0}\right)-J_{1}\left(k r_{0}\right) / r_{0}+i k Y_{0}\left(k r_{0}\right)-\left(i / r_{0}\right) Y_{1}\left(k r_{0}\right)+\left(\nu / r_{0}\right) J_{1}\left(k r_{0}\right)+\left(v / r_{0}\right) i Y_{1}\left(k r_{0}\right)\right]}{H_{0}^{(1)}\left(k r_{0}\right)}\right\}^{-1} \\
& \times\left\{\frac{\left[k J_{0}\left(k r_{0}\right)-J_{1}\left(k r_{0}\right) / r_{0}-i k Y_{0}\left(k r_{0}\right)+\left(i / r_{0}\right) Y_{1}\left(k r_{0}\right)+\left(\nu / r_{0}\right) J_{1}\left(k r_{0}\right)-\left(v / r_{0}\right) i Y_{1}\left(k r_{0}\right)\right]}{H_{0}^{(2)}\left(k r_{0}\right)}\right\},
\end{aligned}
$$




$$
\begin{gathered}
R_{C}=-\left\{\frac{\left[k J_{0}\left(k r_{a}\right)-J_{1}\left(k r_{a}\right) / r_{a}-i k Y_{0}\left(k r_{a}\right)+\left(i / r_{a}\right) Y_{1}\left(k r_{0}\right)+\left(\nu / r_{a}\right) J_{1}\left(k r_{a}\right)-\left(v / r_{a}\right) i Y_{1}\left(k r_{a}\right)\right]}{H_{0}^{(2)}\left(k r_{a}\right)}\right\}^{-1} \\
\cdot\left\{\frac{\left[k J_{0}\left(k r_{a}\right)-J_{1}\left(k r_{a}\right) / r_{a}+i k Y_{0}\left(k r_{a}\right)-\left(i / r_{a}\right) Y_{1}\left(k r_{a}\right)+\left(\nu / r_{a}\right) J_{1}\left(k r_{a}\right)+\left(\nu / r_{a}\right) i Y_{1}\left(k r_{a}\right)\right]}{H_{0}^{(1)}\left(k r_{a}\right)}\right\} .
\end{gathered}
$$

Forward-traveling and negative-traveling waves can be shown clearly in Figure 1. And the expression can be written as follows:

$$
\begin{aligned}
& b_{1}^{+}=f_{1}^{+}\left(r_{a}-r_{0}\right) a_{1}^{+}, \\
& a_{1}^{-}=f_{1}^{-}\left(r_{a}-r_{0}\right) b_{1}^{-} .
\end{aligned}
$$

Considering (17), forward-traveling and negativetraveling waves can be obtained as

$$
\begin{aligned}
& f_{1}^{+}\left(r_{a}-r_{0}\right)=\frac{H_{0}^{(2)}\left(k r_{a}\right)}{H_{0}^{(2)}\left(k r_{0}\right)}, \\
& f_{1}^{-}\left(r_{a}-r_{0}\right)=\frac{H_{0}^{(1)}\left(k r_{0}\right)}{H_{0}^{(1)}\left(k r_{a}\right)} .
\end{aligned}
$$

Similarly, the reflection matrices are (20a) and (24) for case of inner surface fixed and outer surface free.

2.4. Analysis and Discussion. After combining propagation and reflection matrices derived above, the natural characteristics of single circular plate can be analyzed effectively. Hence, it gives

$$
\begin{array}{ll}
b_{1}^{+}=f_{1}^{+}\left(r_{a}-r_{0}\right) a_{1}^{+}, & a_{1}^{+}=R_{A} a_{1}^{-}, \\
a_{1}^{-}=f_{1}^{-}\left(r_{a}-r_{0}\right) b_{1}^{-}, & b_{1}^{-}=R_{C} b_{1}^{+} .
\end{array}
$$

And it yields

$$
F(f)=\left[f_{1}^{+}\left(r_{a}-r_{0}\right) R_{A} f_{1}^{-}\left(r_{a}-r_{0}\right) R_{C}-1\right] b_{1}^{+}=0 .
$$

RESIN is selected as the material of circular plate. Material and structural parameters are tabulated in Table 1.

Substitute parameters listed in Table 1 into (10), (12), (14), and (28), and the natural frequency can be computed. Herein, red and black curves obtained by wave approach are real and imaginary wave solution, respectively. Also, it is important to note that the intersection points of the curves are natural frequency. After solving the inherent characteristic of circular plate structure, the characteristic curves calculated by classical method and wave approach are presented in Figure 2. It can be seen that these natural frequencies intersect at the same point in $x$-axis, which verifies the correctness of the numerical calculation.

\section{Radial Vibration Analysis of Phononic Crystal}

3.1. Model and Transfer Matrix. Figure 3 is the schematic diagram of radial phononic crystal circular plate. Herein, the white region represents RESIN materials and the gray region represents piezoelectric ceramic PZT4. The inner and outer radius are $r_{1}$ and $r_{2}$, respectively. The radial span ratios are denoted with $a_{1}$ and $a_{2}$. And $h$ represents the thickness.

Considering an eight-layer circular plate, it corresponds to the model $A B A B A B A B$. The interfaces between RESIN and PZT4 material are $a, b, c, d, e, f, m, p, q$ (see Figure 3). With regard to RESIN, Young's modulus, density, and Poisson coefficient are $E_{1}, \rho_{1}, \nu_{1}$. For the PZT4, Young's modulus, density, and Poisson coefficient are $E_{2}, \rho_{2}, v_{2}$. When radial wave propagates in RESIN layers $1,3,5$, and 7 , the velocity is $c_{1}=\left[E_{1} / \rho_{1}\left(1-v_{1}^{2}\right)\right]^{1 / 2}$ and the wavenumber is $k_{1}=$ $w / c_{1}=2 \pi f / c_{1}$. The velocity is $c_{2}=\left[E_{2} / \rho_{2}\left(1-v_{2}{ }^{2}\right)\right]^{1 / 2}$ and the wavenumber is $k_{2}=w / c_{2}=2 \pi f / c_{2}$ when wave propagates in PZT4 layers 2, 4, 6, and 8 .

With regard to radial vibration, there are two parameters which satisfy the continuous conditions at the interface of different materials. Namely, they are the radial displacement and radial stress. So transfer matrices of radial vibration can be derived as follows.

At the interface $b\left(r_{b}=r_{1}+a_{1}\right)$, it gives

$$
\begin{aligned}
& u_{1}=u_{2} \\
& \sigma_{1}=\sigma_{2} .
\end{aligned}
$$

Thus, it can be arranged in a matrices form

$$
H_{1}\left[\begin{array}{c}
A_{11}^{+} \\
A_{11}^{-}
\end{array}\right]=K_{1}\left[\begin{array}{c}
B_{12}^{+} \\
B_{12}^{-}
\end{array}\right] .
$$

At the interface $c\left(r_{c}=r_{1}+a_{1}+a_{2}\right)$, it gives

$$
\begin{aligned}
& u_{2}=u_{3}, \\
& \sigma_{2}=\sigma_{3} .
\end{aligned}
$$

Similarly, one has

$$
H_{2}\left[\begin{array}{c}
A_{21}^{+} \\
A_{21}^{-}
\end{array}\right]=K_{2}\left[\begin{array}{l}
B_{12}^{+} \\
B_{12}^{-}
\end{array}\right] .
$$

Combining (30) and (32), one has

$$
\left[\begin{array}{c}
A_{21}^{+} \\
A_{21}^{-}
\end{array}\right]=T_{31}\left[\begin{array}{l}
A_{11}^{+} \\
A_{11}^{-}
\end{array}\right],
$$




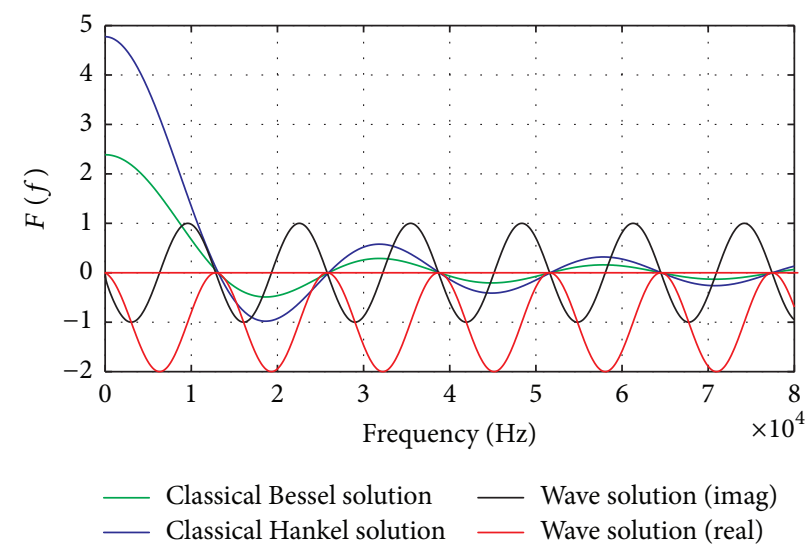

(a) Fixed-fixed boundary

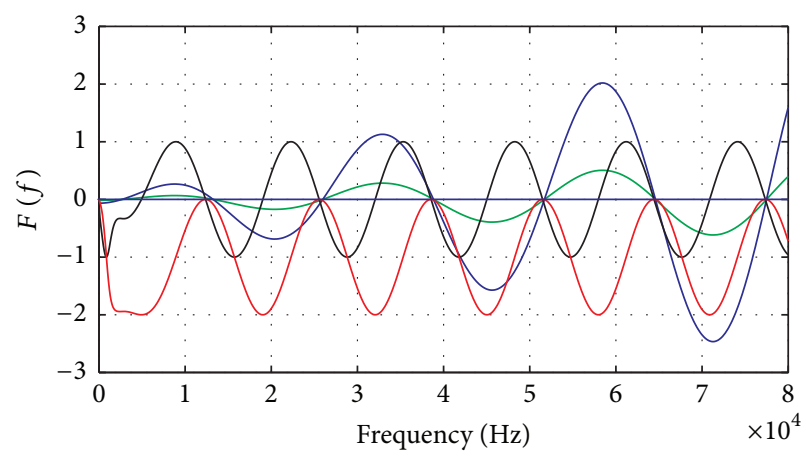

_ Classical Bessel solution _ Wave solution (imag) _ Classical Hankel solution _ Wave solution (real)

(b) Free-free boundary

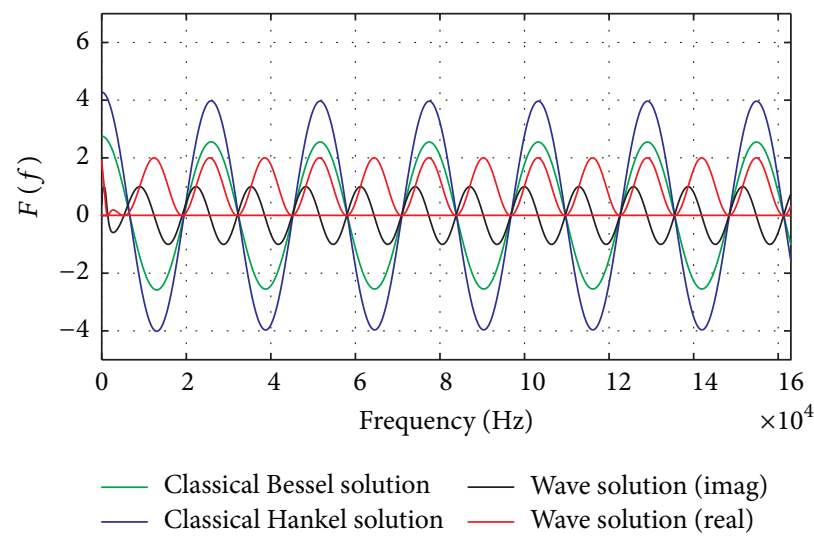

(c) Fixed-free boundary

FIgURE 2: Natural frequency calculated by classic method and wave approach.

TABLE 1: Material and structural parameters.

\begin{tabular}{lccc}
\hline Material parameters & $\rho\left(\mathrm{kg} / \mathrm{m}^{3}\right)$ & $E(\mathrm{~Pa})$ & $v$ \\
RESIN & 1180 & $0.435 \times 10^{10}$ & 0.3679 \\
\hline Structural parameters & $r_{0}(\mathrm{~m})$ & $r_{a}(\mathrm{~m})$ & $h(\mathrm{~m})$ \\
RESIN & 0.08 & 0.16 & 0.005 \\
\hline
\end{tabular}

where $T_{31}=H_{2}^{-1} K_{2} K_{1}^{-1} H_{1}$ is the transfer matrices of radial vibration between the $3 \mathrm{rd}$ and the 1 st ring.

As shown in Figure 3, replace $r_{b}$ and $r_{c}$ (in (30) and (32)) with $r_{d}$ and $r_{e}$. Similarly with $T_{31}$, we can obtain $T_{53}$ which is the transfer matrix between the fifth ring and the third ring.

Similarly, replace $r_{d}$ and $r_{e}$ with $r_{f}$ and $r_{m}$, and the matrix $T_{75}$ can be obtained.

At last, submitting $r_{p}$ into (30), we can get $T_{81}$. Therefore, with regard to the radial phononic crystal, one has

$$
T_{81}=K_{87}^{-1} H_{87} T_{75} T_{53} T_{31} .
$$

3.2. Numerical Result and Discussion. The radial phononic crystal circular plates are composed of two materials, namely, RESIN and piezoelectric ceramic PZT4. Material parameters and structural parameters are consistent with Table 2.
TABLE 2: Material parameters and structural parameters.

\begin{tabular}{lccc}
\hline Material parameters & $\rho\left(\mathrm{kg} / \mathrm{m}^{3}\right)$ & $E(\mathrm{~Pa})$ & $\nu$ \\
\hline I (RESIN) & 1180 & $0.435 \times 10^{10}$ & 0.3679 \\
II (PZT4) & 7500 & $8.13 \times 10^{10}$ & 0.33 \\
\hline Structural parameters & $a_{1}=a_{2}(\mathrm{~m})$ & $r_{2}=2 r_{1}(\mathrm{~m})$ & $h(\mathrm{~m})$ \\
RESIN and PZT4 & 0.01 & 0.16 & 0.003 \\
\hline
\end{tabular}

Additionally, piezoelectric parameters are set as $s_{11}^{E}=$ $12.3 \times 10^{-12} \mathrm{~m}^{2} / \mathrm{N}, \varepsilon_{33}^{T} / \varepsilon_{0}=1300, \varepsilon_{0}=8.84 \times 10^{-12} \mathrm{c} / \mathrm{m}$, and $d_{31}=-123 \times 10^{-12} \mathrm{c} / \mathrm{N}$.

Taking advantage of transfer matrices and boundary conditions, the transmission characteristics are calculated using MATLAB numerically. Figure 4 is the transmission curve of radial vibration with four periodic structures which is composited by single material RESIN and RESIN/PZT4.

Figure 4 is the comparison of numerical and FEM results. It indicates that there is no attenuation when radial wave propagates in a single material, while, for the phononic crystal, the transmission curve shows a big vibration attenuation band after introducing the periodicity. However, when radial wave propagates in the single material, there is no band gap which attenuates weakly and only owns $-4 \mathrm{~dB}$. Furthermore, 

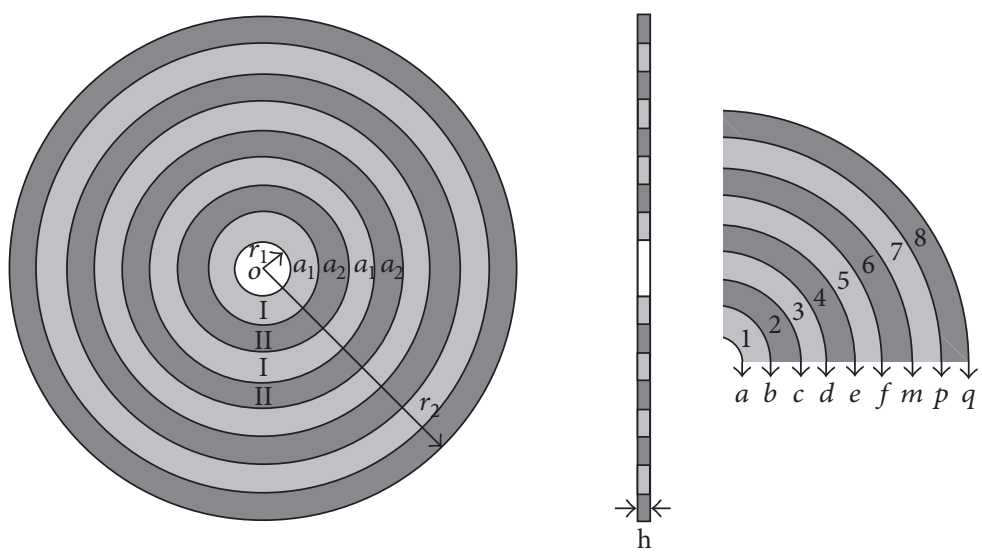

I RESIN

II PZT4

FIGURE 3: Schematic diagram of radial phononic crystal circular plate.

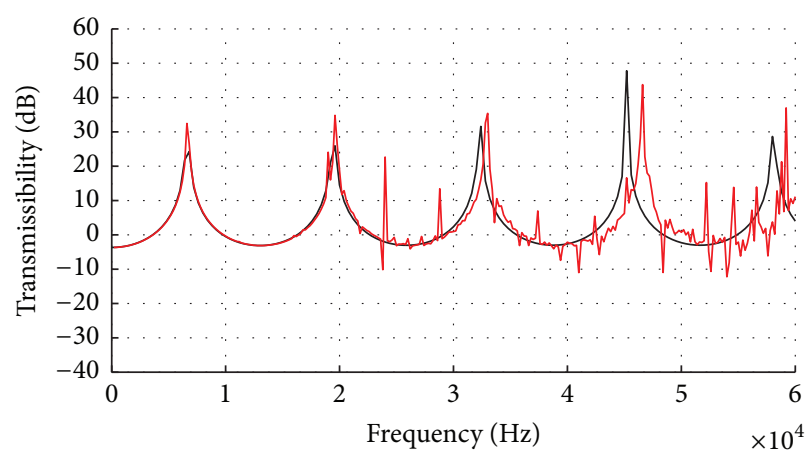

- Numerical result FEM

(a) Single material (RESIN)

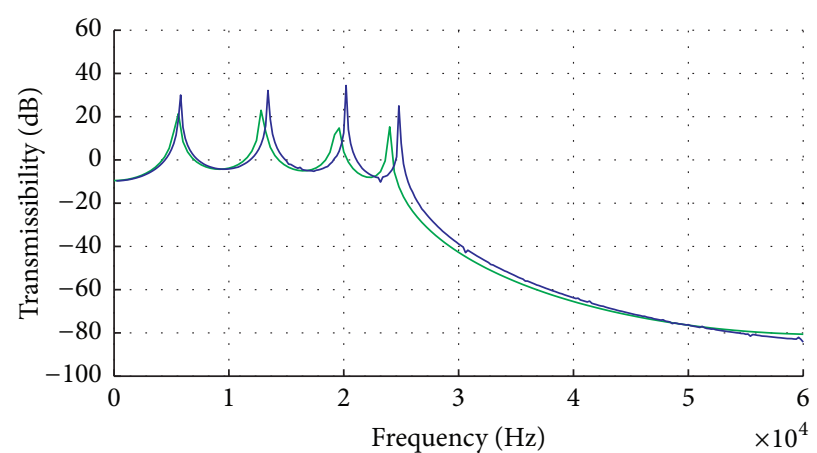

- Numerical result

(b) Phononic crystal (RESIN/PZT4)

FIGURE 4: Radial vibration curves of numerical and FEM results.

ANSYS 14.5 is used to verify the radial propagation characteristics. Using harmonic analysis and keeping the boundary conditions of outer surface free, radial displacement is excited on the inner surface which is shown in Figure 4(b). Comparing numerical results with FEM results, it can be found that the bands are in good agreement with the aspects of position, width, and amplitude attenuation and other aspects, which indicates the correctness of theoretical calculations.

3.3. Effects of Structural Parameters. In this section, the influence of piezoelectric effect on radial wave has been discussed in detail. Furthermore, the influences of piezoelectric parameter $d_{31}$ and polar direction on the band have also been studied in case of open and short circuit for piezoelectric material.

3.3.1. Open and Short Circuit. With regard to piezoelectric material, the $D$-type equation of radial vibration can be expressed as

$$
\varepsilon_{r}=s_{11}^{E} \sigma_{r}+s_{12}^{E} \sigma_{\theta}+d_{31} E_{Z}
$$

$$
\begin{gathered}
\varepsilon_{\theta}=s_{12}^{E} \sigma_{r}+s_{11}^{E} \sigma_{\theta}+d_{31} E_{Z}, \\
D_{z}=d_{31} \sigma_{r}+d_{31} \sigma_{\theta}+\xi_{33}^{T} E_{Z} .
\end{gathered}
$$

It corresponds to short circuit situation when field intensity is $E_{Z}=0$. Therefore, (35) can be simplified as

$$
\begin{aligned}
\sigma_{r} & =\frac{s_{11}^{E}}{\left(s_{11}^{E}\right)^{2}-\left(s_{12}^{E}\right)^{2}} \varepsilon_{r}-\frac{s_{12}^{E}}{\left(s_{11}^{E}\right)^{2}-\left(s_{12}^{E}\right)^{2}} \varepsilon_{\theta}, \\
\sigma_{\theta} & =\frac{s_{11}^{E}}{\left(s_{11}^{E}\right)^{2}-\left(s_{12}^{E}\right)^{2}} \varepsilon_{\theta}-\frac{s_{12}^{E}}{\left(s_{11}^{E}\right)^{2}-\left(s_{12}^{E}\right)^{2}} \varepsilon_{r}, \\
D_{z} & =d_{31}\left(\sigma_{r}+\sigma_{\theta}\right) .
\end{aligned}
$$

Then, it has

$$
\begin{aligned}
\nu^{E} & =-\frac{s_{12}^{E}}{s_{11}^{E}}, \\
Y_{0}^{E} & =\frac{1}{s_{11}^{E}},
\end{aligned}
$$




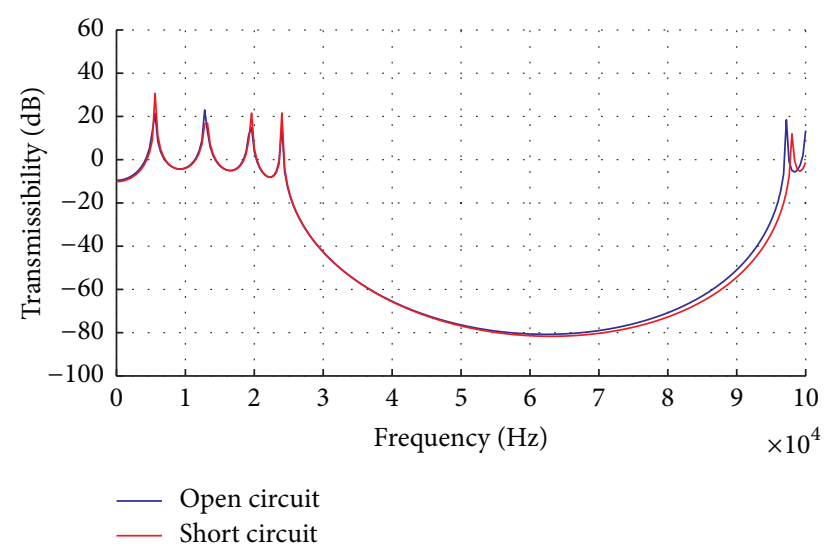

FIGURE 5: Comparison diagram with short and open circuits.

where $\nu^{E}$ denotes Poisson's coefficient in the condition of short circuit. $Y_{0}^{E}$ denotes Young modulus.

Piezoelectric material is in the open circuit when the charge is $D_{z}=0$. Thus, (35) can be simplified as

$$
\begin{gathered}
\sigma_{r}=\frac{Y^{D}}{1-\left(\nu^{D}\right)^{2}}\left(\varepsilon_{r}+\nu^{D} \varepsilon_{\theta}\right), \\
\sigma_{\theta}=\frac{Y^{D}}{1-\left(\nu^{D}\right)^{2}}\left(\varepsilon_{\theta}+\nu^{D} \varepsilon_{r}\right) .
\end{gathered}
$$

Then, it gives

$$
\begin{aligned}
\nu^{D} & =-\frac{\xi_{33}^{T} s_{12}^{E}-d_{31}^{2}}{\xi_{33}^{T} s_{11}^{E}-d_{31}^{2}}, \\
Y_{0}^{D} & =\frac{1}{s_{11}^{E}-d_{31}^{2} / \xi_{33}^{T}},
\end{aligned}
$$

where $v^{D}$ denotes Poisson's coefficient in the condition of open circuit. $Y_{0}^{D}$ denotes Young modulus.

By using transfer matrices derived in Section 2.1, vibration characteristics curves have been calculated for the case of short and open circuit. As depicted in Figure 5, the selected frequency band is $0-10 \mathrm{kHz}$. It indicates that the band changes obviously in the high frequency because of the piezoelectric effect. However, the short circuit curve moves toward low frequency slightly. From the above theoretical analysis by comparing (37) and (39), it can be observed that the elasticity modulus and Poisson's coefficient of piezoelectric materials are different when they are in short or open circuit. For the case of open circuit, elasticity modulus is larger. Meanwhile, Poisson's coefficient also changes. It is important to note that these two parameters can influence the value of $\sigma_{r}$ and $\sigma_{\theta}$ directly, which means that radial wavenumber changes. Thus, it is the natural mechanism of band moving.

3.3.2. Piezoelectric Parameters and Inner Radius. As to the open circuit case, the effects of electrical parameter $d_{31}$ and inner radius $r_{0}$ on radial wave band are depicted in Figure 6. Herein, Figure 6(a) describes the effect of piezoelectric

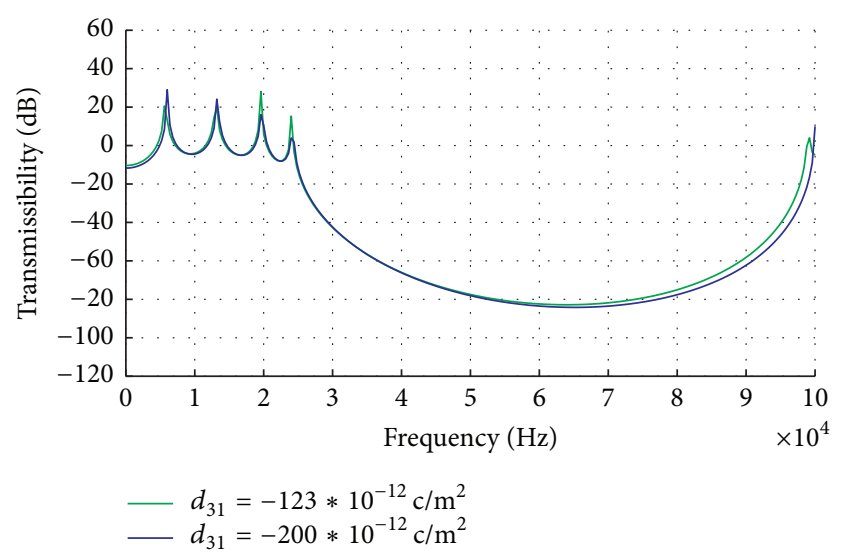

(a) Effect of piezoelectric parameter $d_{31}$

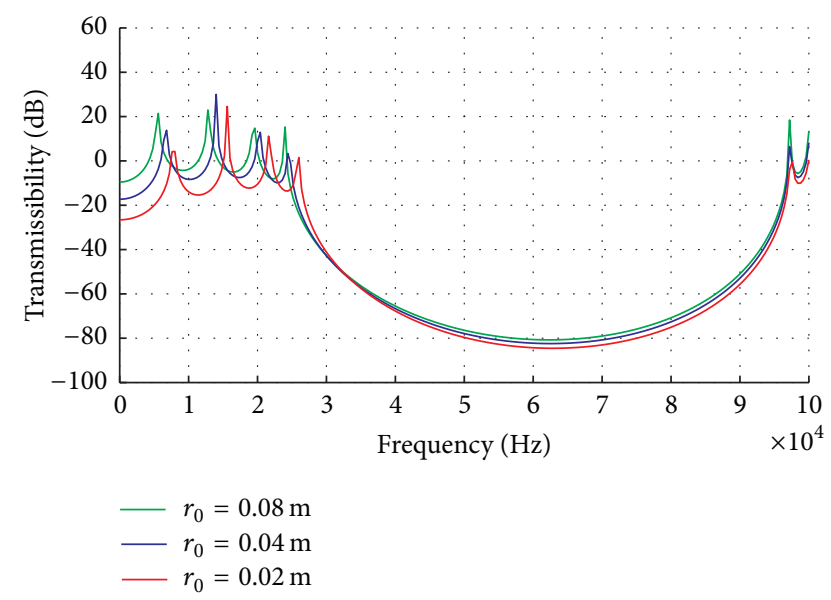

(b) Effect of inner radius $r_{0}$

FIGURE 6: Effect of piezoelectric modulus $d_{31}$ and inner radius $r_{0}$.

parameter $d_{31}$. However, Figure 6(b) displays the effect of inner radius $r_{0}$.

From (39), it can be easily observed that $d_{31}$ determines the values of $Y_{0}^{D}$ and $v^{D}$ which can influence the band position and attenuation. Figure 6(a) indicates that radial band has been expanded with the increase of $d_{31}$ (here, the negative sign only represents direction). However, the change is not obvious. Figure 6(b) displays that inner radius $r_{0}$ can influence the low frequency greatly. It is also clear from the figure that the band attenuates obviously at low frequency. However, the effect on high frequency is much smaller.

\section{Conclusion}

This paper presents the classical method and wave propagation approach to obtain the natural frequency. Based on radial vibration equation, propagation and reflection matrices are derived. It can be found that natural frequency of the single circular plate can be calculated conveniently by using wave propagation approach. Then, the results obtained by using wave approach are compared with the classical method at the boundary conditions of free-free, fixed-fixed, and fixed-free. 
It can be seen that the results calculated by these two methods coincide with each other.

Additionally, the band phenomenon of radial phonon crystal circular plate is also analyzed for a deeper understanding of radial vibration. FEM is used to verify the theoretical results. It can be found that the inner radius of circular plate has great influence on the low frequency. Piezoelectric coefficient, open circuit, and short circuit make the elastic modulus and Poisson's ratio change. Then the radial wavenumber also changes, which is the natural mechanism of band removing for the case of open and short circuits.

\section{Competing Interests}

The authors declare that they have no competing interests.

\section{Acknowledgments}

This work is financially supported by the National Natural Science Foundation of China (no. 51375105) and the Natural Science Foundation of Heilongjiang Province of China (E201418).

\section{References}

[1] B. R. Mace, "Wave reflection and transmission in beams," Journal of Sound and Vibration, vol. 97, no. 2, pp. 237-246, 1984.

[2] C. Mei and B. R. Mace, "Wave reflection and transmission in Timoshenko beams and wave analysis of Timoshenko beam structures," Journal of Sound and Acoustics, vol. 127, pp. 382-394, 2004.

[3] N. R. Harland, B. R. Mace, and R. W. Jones, "Wave propagation, reflection and transmission in tunable fluid-filled beams," Journal of Sound and Vibration, vol. 241, no. 5, pp. 735-754, 2001.

[4] B. Kang, C. H. Riedel, and C. A. Tan, "Free vibration analysis of planar curved beams by wave propagation," Journal of Sound and Vibration, vol. 260, no. 1, pp. 19-44, 2003.

[5] S.-K. Lee, B. R. Mace, and M. J. Brennan, "Wave propagation, reflection and transmission in curved beams," Journal of Sound and Vibration, vol. 306, no. 3-5, pp. 636-656, 2007.

[6] S. K. Lee, Wave reflection, transmission and propagation in structural waveguides [Ph.D. thesis], Southampton University, 2006.

[7] C. H. Wang and L. R. F. Rose, "Wave reflection and transmission in beams containing delamination and inhomogeneity," Journal of Sound and Vibration, vol. 264, no. 4, pp. 851-872, 2003.

[8] C. A. Tan and B. Kang, "Wave reflection and transmission in an axially strained, rotating Timoshenko shaft," Journal of Sound and Vibration, vol. 213, no. 3, pp. 483-510, 1998.

[9] X. B. Li, "Study on free vibration analysis of circular cylindrical shells using wave propagation," Journal of Sound and Vibration, vol. 311, no. 3-5, pp. 667-682, 2008.

[10] L. Gan, X. B. Li, and Z. Zhang, "Free vibration analysis of ringstiffened cylindrical shells using wave propagation approach," Journal of Sound and Vibration, vol. 326, no. 3-5, pp. 633-646, 2009.

[11] X. M. Zhang, "Vibration analysis of cross-ply laminated composite cylindrical shells using the wave propagation approach," Applied Acoustics, vol. 62, no. 11, pp. 1221-1228, 2001.
[12] X. M. Zhang, "Frequency analysis of submerged cylindrical shells with the wave propagation approach," International Journal of Mechanical Sciences, vol. 44, no. 7, pp. 1259-1273, 2002.

[13] M. Shakeri, M. Akhlaghi, and S. M. Hoseini, "Vibration and radial wave propagation velocity in functionally graded thick hollow cylinder," Composite Structures, vol. 76, no. 1-2, pp. 174181, 2006.

[14] D. Torrent and J. Sánchez-Dehesa, "Acoustic resonances in twodimensional radial sonic crystal shells," New Journal of Physics, vol. 12, Article ID 073034, 2010.

[15] J. Carbonell, D. Torrent, and J. Sanchez-Dehesa, "Radial photonic crystal shells and their application as resonant and radiating elements," IEEE Transactions on Antennas and Propagation, vol. 61, no. 2, pp. 755-767, 2013.

[16] Z. L. Xu, F. G. Wu, and Z. N. Guo, "Low frequency phononic band structures in two-dimensional arc-shaped phononic crystals," Physics Letters A, vol. 376, no. 33, pp. 2256-2263, 2012.

[17] T. Ma, T. N. Chen, X. P. Wang, Y. Li, and P. Wang, "Band structures of bilayer radial phononic crystal plate with crystal gliding," Journal of Applied Physics, vol. 116, no. 10, Article ID 104505, pp. 1-7, 2014.

[18] H. S. Shu, L. Q. Dong, S. D. Li et al., "Propagation of torsional waves in a thin circular plate of generalized phononic crystals," Journal of Physics D: Applied Physics, vol. 47, no. 29, Article ID 295501, 2014.

[19] H. S. Shu, W. Liu, S. D. Li et al., "Research on flexural wave band gap of a thin circular plate of piezoelectric radial phononic crystals," Journal of Vibration and Control, vol. 22, no. 7, pp. 1777-1789, 2016.

[20] K. Kiani, "Elasto-dynamic analysis of spinning nanodisks via a surface energy-based model," Journal of Physics D: Applied Physics, vol. 49, no. 27, Article ID 275306, 2016.

[21] K. Kiani, "Thermo-mechanical analysis of functionally graded plate-like nanorotors: a surface elasticity model," International Journal of Mechanical Sciences, vol. 106, pp. 39-49, 2016.

[22] K. Kiani, "Magneto-elasto-dynamic analysis of an elastically confined conducting nanowire due to an axial magnetic shock," Physics Letters A, vol. 376, no. 20, pp. 1679-1685, 2012.

[23] S. Y. Lin, "Study on the step-type circular ring ultrasonic concentrator in radial vibration," Ultrasonics, vol. 49, no. 2, pp. 206-211, 2009. 


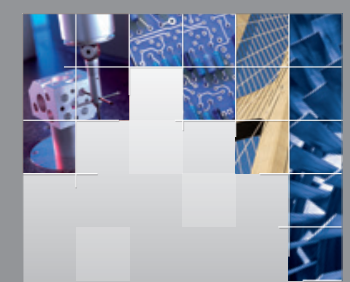

\section{Enfincering}
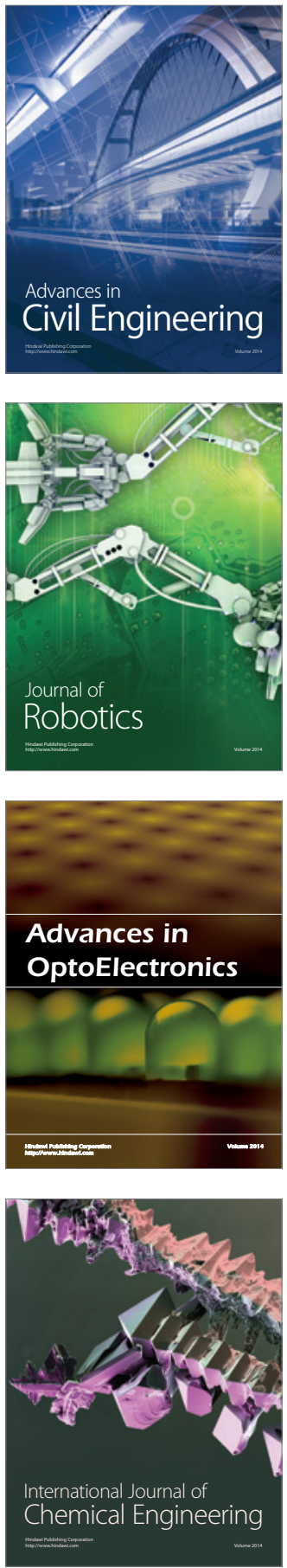

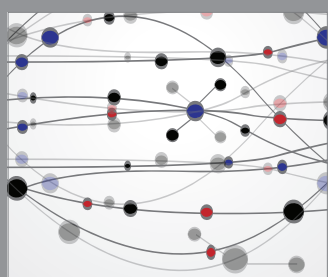

The Scientific World Journal

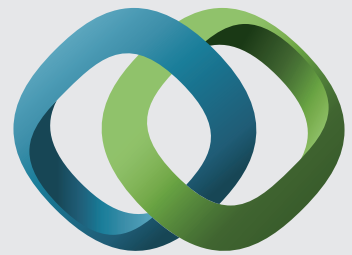

\section{Hindawi}

Submit your manuscripts at

http://www.hindawi.com
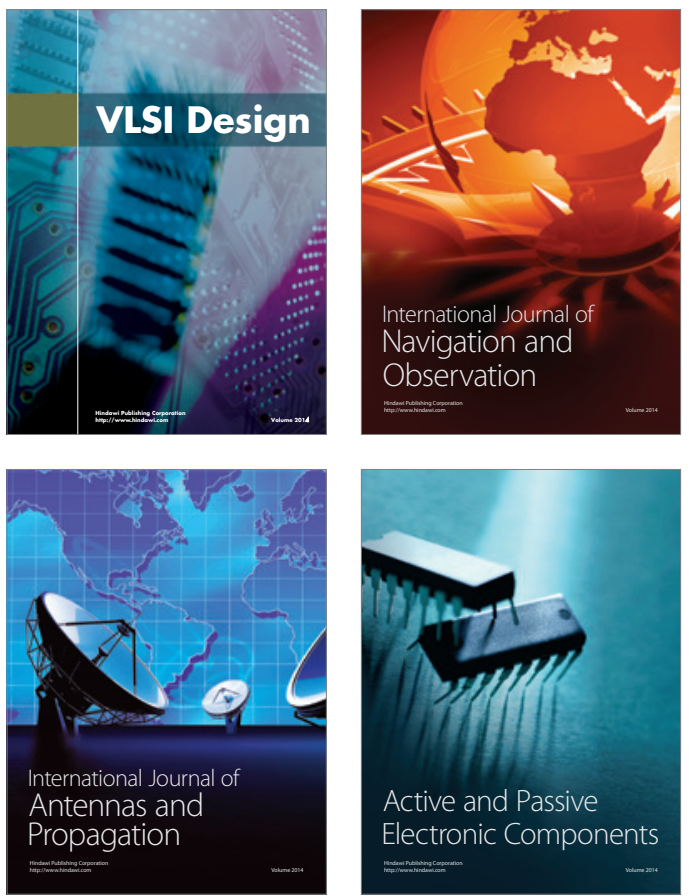
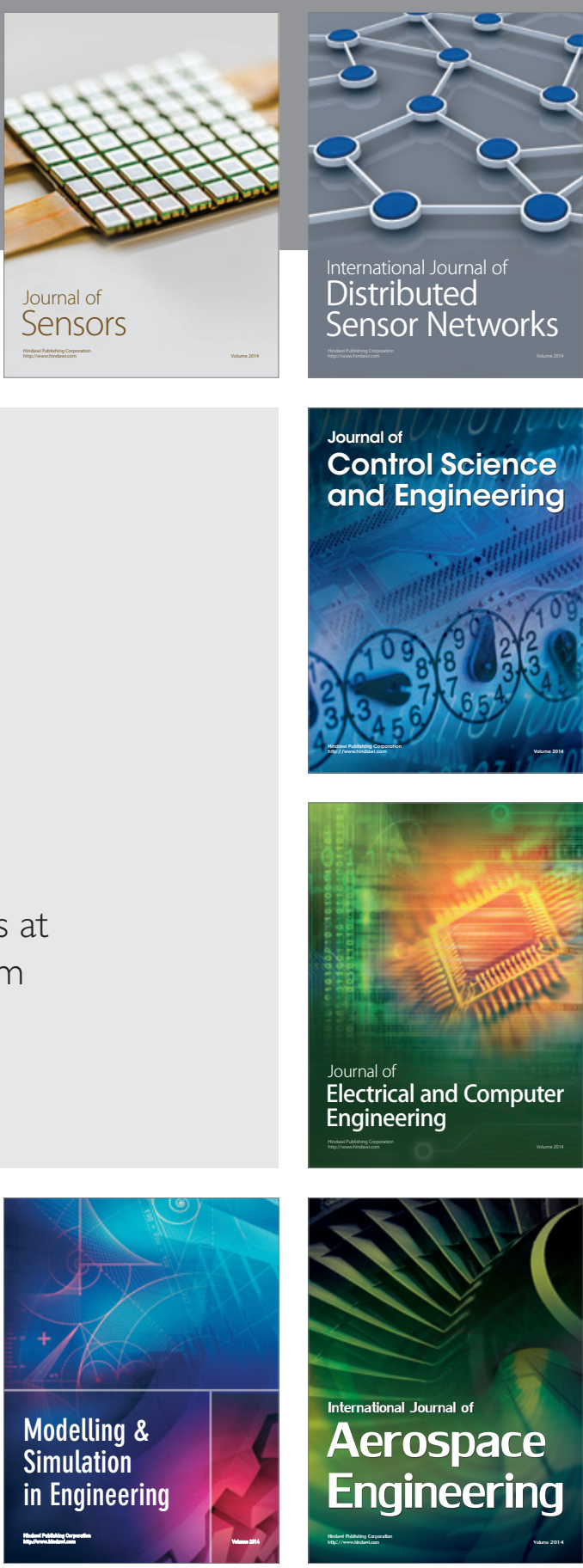

International Journal of

Distributed

Sensor Networks

Journal of

Control Science

and Engineering
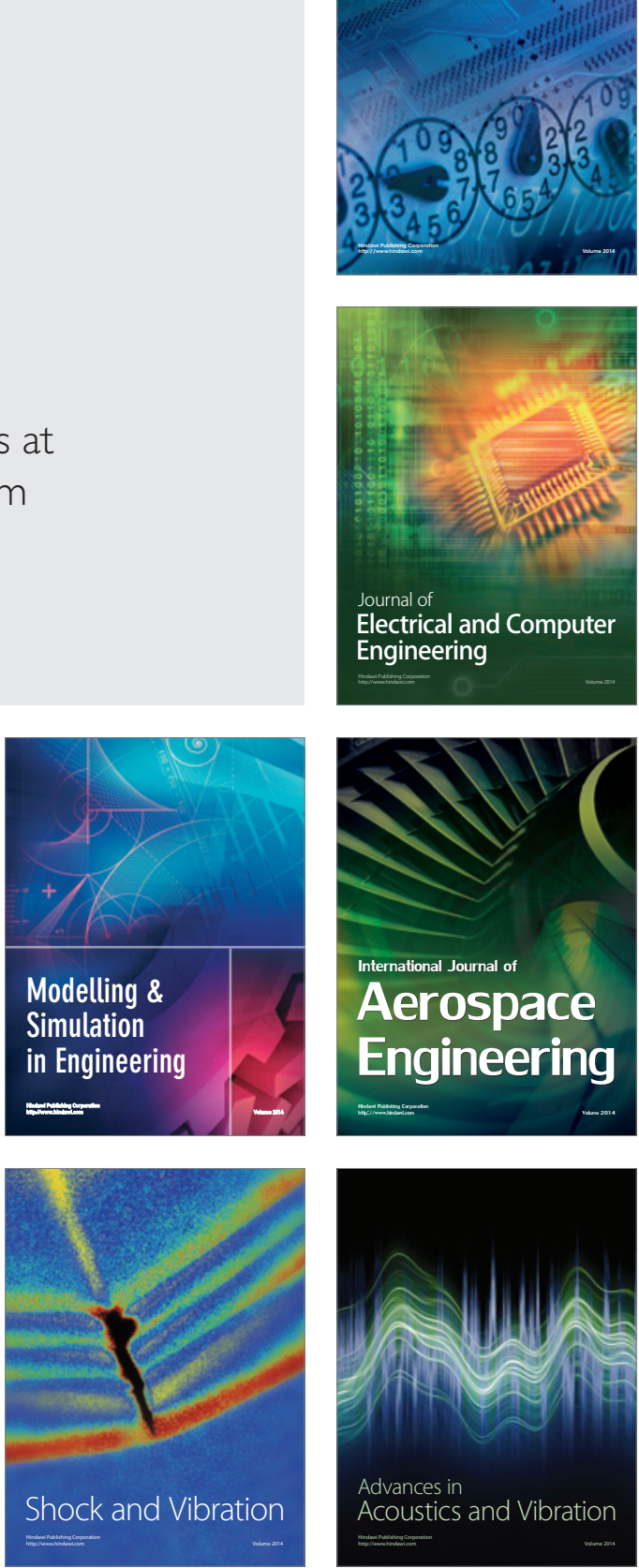